Stenroos, Matti; Hunold, Alexander; Eichardt, Roland; Haueisen, Jens:

Comparison of three- and single-shell volume conductor models in magnetoencephalography

Zuerst erschienen in: Biomedical Engineering = Biomedizinische Technik. - Berlin [u.a.] : de Gruyter. - 57 (2012), Suppl. 1, Track-M, p. 311.

Erstveröffentlichung: $\quad$ 2012-09-04

ISSN (online): $\quad$ 1862-278X

ISSN (print): $\quad$ 0013-5585

DOI: $\quad 10.1515 / \mathrm{bmt}-2012-4396$

[Zuletzt gesehen: 2019-08-15]

„Im Rahmen der hochschulweiten Open-Access-Strategie für die Zweitveröffentlichung identifiziert durch die Universitätsbibliothek IImenau. “

"Within the academic Open Access Strategy identified for deposition by Ilmenau University Library."

„Dieser Beitrag ist mit Zustimmung des Rechteinhabers aufgrund einer (DFGgeförderten) Allianz- bzw. Nationallizenz frei zugänglich."

"This publication is with permission of the rights owner freely accessible due to an Alliance licence and a national licence (funded by the DFG, German

DFG

Research Foundation) respectively." 


\section{Comparison of three- and single-shell volume conductor models in magnetoencephalography}

M. Stenroos, Department of Biomedical Engineering and Computational Science, Aalto University, Espoo, Finland / MRC Cognition and Brain Sciences Unit, Cambridge, England, matti.stenroos@aalto.fi

A. Hunold, Institute of Biomedical Engineering and Informatics, Technical University Ilmenau, Germany, alexander.hunold@tu-ilmenau.de

R. Eichardt, Institute of Biomedical Engineering and Informatics, Technical University Ilmenau, Germany, roland.eichardt@tu-ilmenau.de

J. Haueisen, Institute of Biomedical Engineering and Informatics, Technical University Ilmenau, Germany, jens.haueisen@tu-ilmenau.de

\section{Introduction}

In experimental magnetoencephalography (MEG), the head is commonly modelled as homogeneous volume conductor, shaped according to the inner skull boundary. In this 1-shell model, the skull is thus assumed a perfect insulator. In the 3-shell model, also the skull and scalp are modelled. In this work, we analyze the error generated by omitting the skull and scalp and compare it to the variation between different 3 -shell models.

\section{Methods}

A surface-based head model was generated from T1 and FLASH MR images: conductivity boundaries were segmented with MNE-Suite, Curry, and FieldTrip programs, and the cortical source region was segmented with FreeSurfer. MNESuite used both MR sets, while other programs used the T1 set only. The meshes were co-registered with the sensor surface of the 306-channel Elekta MEG system. Lead field matrices (LFM) for 3- and 1-shell models were built using the Quick Galerkin boundary-element method and compared using standard metrics.

\section{Results}

The anatomical model produced with MNE-Suite was smoothest and had the most regular triangulation. The FieldTrip model added some detail. The Curry model had thinner skull that was in some regions farther away from the sources, and the head was truncated below the brain.

Comparing different 3-shell models, mean relative morphological differences (RDM) and magnitude ratios (MAG) were between $0.13-0.18$ and 0.96-1.07, respectively. Corresponding measures between 3- and 1-shell models were between $0.17-0.37$ and $0.99-1.11$. The largest differences were obtained in the temporal lobe and sylvian fissure. In comparis on between the segmentation tools, Curry-based LFMs produced the largest differences.

\section{Conclusion}

The results show that the differences between 3-shell models built with different segmentation tools are considerably smaller than the errors due to the 1-shell simplification. As the effort of constructing 3-and 1-shell models is comparable, we recommend the more realistic 3-shell model. 